\title{
EMPIRICAL RELATIONSHIP BETWEEN INLET CROSS-SECTIONAL AREA AND TIDAL PRISM: A RE-EVALUATION
}

\author{
Marcel J.F. Stive ${ }^{1}$, Liang Ji ${ }^{1}$, Ronald L. Brouwer ${ }^{1}$, J. (Co) van de Kreeke $^{2}$ and Roshanka \\ Ranasinghe ${ }^{1,3}$
}

\begin{abstract}
The well-known empirical relationship between the equilibrium cross-sectional area of tidal inlet entrances $(A)$ and the tidal prism $(P)$, first developed by O'Brien (1931), has been extensively reviewed. Our theoretical investigations indicate that a unique $A-P$ relationship should only be expected for clusters of inlets that are phenomenological similar (i.e. fairly similar hydrodynamic and morphological conditions), and that the exponent $q$ in the $A-P$ relation should be larger than 1 . However, relevant published data available to date do not clearly support this theoretical finding. A re-analysis of the available data sets by Stive et al. (2009) indicated that they may not be sufficiently reliable to verify our theoretical finding with regard to $q>1$ due to the violation of the condition of phenomenological similarity, and possibly also due to violating the initial definitions given by O'Brien (1931) in estimating the tidal prism. The resolution of this issue is important because slightly different values of $q$ result in significantly variable values for the equilibrium cross-sectional area of the tidal entrance. This may have significant implications in determining the true stable equilibrium entrance cross-sectional area. Here we present a re-analysis of the available data with a focus on determining the phenomenological dependencies of the $A-P$ relationship. The available $A-P$ data from the US Pacific, Atlantic and Gulf coasts (Jarrett, 1976 and Powell, 2003) have been re-scrutinized and categorized following the above mentioned phenomenological similarity criteria, viz. similar tidal range, similar sediment size, similar littoral transport and similar hydraulic radius. All together, some 20 different categories were considered and $A-P$ relationships were obtained for each category. Generally, high correlations were found between the stable inlet predicted by each $A-P$ relationship and the corresponding data. However, only in a limited number of categories were they significantly better than the correlations for the complete datasets. Finally, we point out that only in a number of categories the $q$ value associated with the A-P relationship exceeded unity as suggested by the theoretical derivations. In the majority of categories the $q$ value associated with the $A-P$ relationship does not exceed unity. This is truly disappointing, and we have no physical explanation for this and consider this issue unresolved.
\end{abstract}

Keywords: inlets, tidal prism, empirical relationship, equilibrium cross-sectional area

\section{INTRODUCTION}

Tidal inlet and estuary entrances around the world that are in a stable morphological equilibrium state appear to obey a consistent relationship between their gorge cross-sectional area and a characteristic gorge flow parameter. Probably, the first relevant reference in this context is LeConte (1905), who, based on observations of a small number of inlet entrances and harbours on the Pacific Coast of the US, found an empirical relationship between the inlet cross-sectional area and the tidal prism. The pioneering work of LeConte (1905) was then followed up by O'Brien (1931), O'Brien (1969) and Jarrett (1976). The general form of the empirical relationship for the equilibrium crosssection based on the tidal prism is as follows:

$$
A=C P^{q}
$$

where $A\left(\mathrm{~m}^{2}\right)$ is the cross-sectional area (relative to mean sea level) and $P\left(\mathrm{~m}^{3}\right)$ is generally the spring tidal prism. $C$ (generally dimensional!) and $q$ (always dimensionless!) are empirical parameters obtained from observational data. As in the above references we limit our review to inlets that are scoured in a sandy environment, are filled in by littoral sand transport and have negligible freshwater sources.

A selected overview of currently available literature presenting values for the empirical parameters $C$ and $q$ include O'Brien (1969), Jarrett (1976), Van de Kreeke (1992), Powell et al. (2006) for US inlets, Shigemura (1980) for Japanese inlets, Dieckmann et al.(1988) for Wadden Sea inlets, Gerritsen et al. (1990) and Van de Kreeke (1998) for Dutch inlets and Hume and Herdendorf (1993) for New Zealand inlets. As we will argue later, we object to the clustering of data as done in several of these references for inlets that are not in similar tidal, wave and morphological environments. Our arguments

\footnotetext{
${ }^{1}$ Faculty of Civil Engineering \& Geosciences, Delft University of Technology, Stevinweg 1, 2628 CN Delft, NL

${ }^{2}$ Rosenstiel School of Marine and Atmospheric Science, University of Miami, 4600 Rickenbacker Causeway, Miami, 33149 USA

${ }^{3}$ Department of Water Engineering, UNESCO-IHE, PO Box 3015, 2601 DA Delft, The Netherlands
} 
are based on our overview of the literature in the next section that suggests a theoretical background for the empirical relationship (equation (1)). In section 3 we will then review and comment upon the observations. Section 4 presents the re-scrutiny of the data. The last section presents our conclusions (section 5).

\section{THEORETICAL DERIVATIONS OF THE A-P RELATIONSHIP}

More recently, after the pioneering, largely empirical work of LeConte (1905) and O'Brien (1931, 1969), theoretical backgrounds of the empirical $A-P$ relationship have been suggested in the literature. The Escoffier diagram (1940) enters this discussion, although this is not obvious. We will review a range of theoretical approaches and use the Escoffier diagram as a starting point, because of its didactic value, i.e. this author introduces the concept of morphological equilibrium.

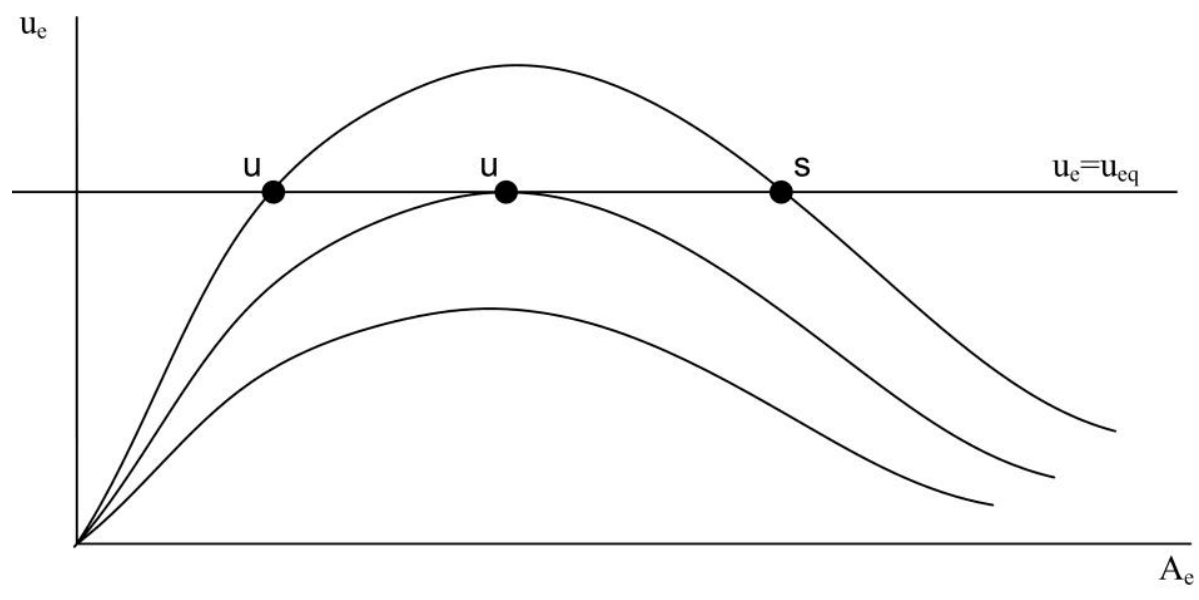

Figure 1. Escoffier diagram expressing the amplitude of the entrance velocity $u_{e}$ as a function of the entrance cross-section $A_{e}$ (Escoffier's Figures 1, 2 and 3 suggesting three possible closure curves and the equilibrium curve $u_{\text {eq }} ; U$ indicates in the wording of Escoffier stationary but unstable and $S$ stationary and stable conditions ${ }^{4}$ ).

For a given inlet, the Escoffier diagram expresses the variation of the maximum velocity in the inlet entrance, $u_{e}$, with the subscript indicating the entrance or gorge, as a function of the cross-section of the gorge $A_{e}$ (Figure 1). This curve can be constructed by calculating the inlet entrance velocity $u_{e}$ by varying the channel area by an analytical or numerical model. The straight line indicated as $u_{e q}$ is the stability curve (equilibrium velocity curve) introduced by Escoffier (1940). He introduced three possible closure curves relative to the stability criterion (see Figure 1, where the three different closure curves refer to possible curves valid for inlets that are under the impact of human interventions or natural hazards), and explained that while all intersections in Figure 1 lead to a equilibrium situation (that may theoretically fulfil the $A-P$ relationship) the only stable equilibrium is the right hand intersection of the top closure curve and the equilibrium velocity curve. We conjecture that this situation is what the $A-P$ relation is referring to.

The stability curve (equilibrium velocity curve) that Escoffier introduced assumes that the equilibrium velocity, $u_{e q}$, is a constant that depends only on the sediment diameter, and suggests that a good, average approximation of the velocity is $3 \mathrm{feet} / \mathrm{second}(0.9 \mathrm{~m} / \mathrm{s})$. His assumption has the following consequence. If as a first approximation we assume a sinusoidal tidal motion, we can relate the maximum entrance velocity to the tidal prism (this can be achieved by using an analytical or numerical model, the outcome of which may vary somewhat dependent on the degree of schematization adopted by such model):

4 Escoffier (1940) introduces the term stationary for these intersections, meaning that these intersections theoretically refer to equilibrium. We find this confusing, because stationary refers usually to temporal stationarity. Our interpretation is that an equilibrium condition is dynamic, i.e. it oscillates around this equilibrium and as soon as it oscillates and thus defers from its value it is either stable or unstable. We thus prefer using the terminology stable or unstable equilibrium. 


$$
u_{e}=\frac{\pi P}{A T}
$$

with $T$ being the tidal period. For equilibrium conditions, $u_{e}=u_{e q}=$ constant this implies that in equation (1) $q=1$. Furthermore, assuming a semi-diurnal tide with $T=44,700 \mathrm{~s}, u_{e q}=0.9 \mathrm{~m} / \mathrm{s}$ results in $C=7.810^{-5}\left(\mathrm{~m}^{-1}\right)$. This is the order of magnitude value for $C$ found empirically for the $A-P$ relationship when $q=l$ (see next section).

However, we have strong evidence from literature (cf. Van de Kreeke, 1985, 1990) to claim that $u_{e}$ is a function of $A_{e}$, implying that $q$ is not equal to 1 (which was shown to have large consequences for the theoretical equilibrium cross-section in Stive et al., 2009). This arises when we adopt the oftenposed and physically well-based concept that the equilibrium area of an inlet is determined by the balance between the transporting ebb-tidal capacity of the entrance flow and the littoral or alongshore transport. We reference here LeConte (1905), O'Brien (1931 and 1969), Riedel and Gourlay (1980), Hume and Herdendorf (1990), Van de Kreeke (1992), Kraus (1998) and Van de Kreeke (2004). The latter reference contains the essentials of most relevant earlier references and we will follow the argumentations here.

The sediment that is transported to the inlet entrance by wave-induced alongshore currents is carried to the inlet by the flood tidal currents. When the inlet is in equilibrium, this sediment is transported back in seaward direction by the ebb-tidal currents. The annual mean flux of sand entering the inlet on the flood is $M\left(\mathrm{~m}^{3} / \mathrm{s}\right) . M$ is assumed a constant fraction of the annual mean alongshore sediment transport and is assumed to remain constant in time. The remaining fraction is assumed to bypass the inlet via the ebb-tidal delta. When in equilibrium, the annual mean flux $M$ equals the annual mean ebb-tidal sediment flux.

The ebb-tidal sediment transport in the entrance $T R$ is taken proportional to the power $n$ of the velocity amplitude and the power $m$ of a length dimension of the cross-section $l$ :

$$
T R=k u_{e}^{n} l^{m}
$$

The subscript $e$ refers to the entrance section. The coefficient $k$ is a constant and its value is dependent on the sediment characteristics. The values of $n$ and $m$ depend on the adopted sediment transport formulation, where $n$ is in the range 3 to 6 and $m$ of order 1 and the length scale $l$ is either the annually averaged width or depth, the final choice depending on the way sediment enters and leaves the inlet entrance (Van de Kreeke, 1992; van de Kreeke, 2004). In the following we will adopt the assumption that for a given offshore tide $u_{e}$ is a unique function of the entrance cross-sectional area $A_{e}$.

After a change in the entrance cross-sectional area, the shape of the cross-section is assumed to remain geometrically similar. This allows expressing the length scale as a constant $\alpha$ times the square root of the annual mean cross-sectional area $A_{e}$ :

$$
l=\alpha \sqrt{A_{e}}
$$

The value of $\alpha$ depends on the shape of the cross-section and the definition of $l$. Substituting the expression (4) for $l$ results in an expression for the annual mean ebb tidal sediment transport as function of the cross-sectional area:

$$
T R=k \alpha^{m} u_{e}^{n} A_{e}^{m / 2}
$$

In this equation $u_{e}$ is a function of $A_{e}$. When the cross-section is in equilibrium:

$$
T R=M
$$

The shapes of the functions, which are independent for realistic values of the parameters $k, \alpha$ and $m$, represented by equations (5) and (6) are plotted in Figure 2a. In general, there will be two values of the cross-sectional area for which the annual mean ebb tidal transport equals the mean annual influx of sediment $M\left(A_{e q 1}\right.$ and $\left.A_{e q 2}\right)$. 
A difficulty in determining the value of $A_{e q 1}$ and $A_{e q 2}$ from equations (5) and (6) is ascertaining the values $k, n, m$ and $\alpha$ for a given inlet situation with a given mean annual influx of sediment $M$. Kraus (1998) undertakes such an approach and arrives at an $A-P$ relationship with a power $q$ of 0.9 and the coefficient a function of the mentioned parameters but also of the width and the depth of the inlet entrance. This implies that the coefficient is a function of $A_{e}$, so that the power $q$ is not 0.9 when we remove the dependence of $A_{e}$, as is assumed commonly. We will further discuss this below.

An elegant way to circumvent ascertaining the parameters was followed by Van de Kreeke (2004) who uses the cross-sectional area versus tidal prism relationship (equation 2) for inlets at equilibrium. From equations (2), (5) and (6) it follows that for inlets at equilibrium:

$$
A_{e}=A_{e q}
$$

with

$$
A_{e}=C P^{q}
$$

and

$$
\begin{gathered}
C=\left(\frac{M T^{n}}{k \alpha^{m} \pi^{n}}\right)^{\left(\frac{2}{m-2 n}\right)} \\
q=\frac{n}{n-m / 2}
\end{gathered}
$$

Equation (7) implies that for a set of inlets in equilibrium that have the same values of $M, T, k, \alpha, m$ and $n$ values of $C$ and $q$ theoretically should be the same. We will address this further in the next section.

We now reintroduce Escoffier's equilibrium velocity $u_{e q}$. Eliminating $P$ between equations (2) and (7) for inlets at equilibrium

$$
u_{e}=u_{e q}
$$

with

$$
u_{e q}=\pi C^{-1 / q} A_{e}^{-1+1 / q} T^{-1}
$$

With values of $q$ close to $1, u_{e q}$ is a weak function of $A_{e}$. The relationship between velocity amplitude and entrance cross-sectional area can be formally expressed as

$$
u_{e}\left(A_{e}\right)=f\left(A_{e} ; A_{b}, \alpha, L_{e}, F, \eta_{o}, T\right)
$$

with the parameters $A_{b}=$ basin surface area, $L_{e}=$ length of entrance section, $F=$ friction factor, $\eta_{0}=$ ocean tide amplitude and $T=$ tidal period.

The general shape of equations (8) and (9) is presented in Figure $2 \mathrm{~b}$. In plotting equation (8), it is assumed that $q>1$. For $q>1$, equation (8) implies that $u_{e q}$ is a decreasing function of $A_{e q}$. This is a direct consequence of equation (3) in which it is assumed that the ebb-tidal transport increases with a length dimension of the inlet, e.g. the width and thus the cross-sectional area, which we assume is physically most realistic. The larger the entrance is, the easier it is to flush out the sediment. For $q=1 u_{e q}$ is a straight line and for $q<1 u_{e q}$ increases monotonously. The roots $A_{e q 1}$ and $A_{e q 2}$ of equations (8) and (9) are the same as those of equations (5) and (6) and equations (7) and (8). The root $A_{\text {eq2 }}$ is, following Escoffier (1940), the stable equilibrium cross-section which the A-P relationship corresponds to.

Starting from physical principles, several investigators have attempted to derive the value of the exponent $q$ from theory. Our analysis of the literature suggests that three approaches are sound and exemplary for other attempts. Yanez (1989) as summarized by Van de Kreeke (1992) took the ebb tidal sediment transport proportional to the power $n$ of the velocity amplitude and the width of the entrance section, $w$ :

$$
T R=k u_{e}^{n} w
$$


Comparing to equation (3), it follows $l=w$ and $m=1$. Substituting $m=1$ in the expression for $q$ (equation 7) and varying $n$ between 3 and 5, the value of $q$ varies between 1.11 and 1.20.

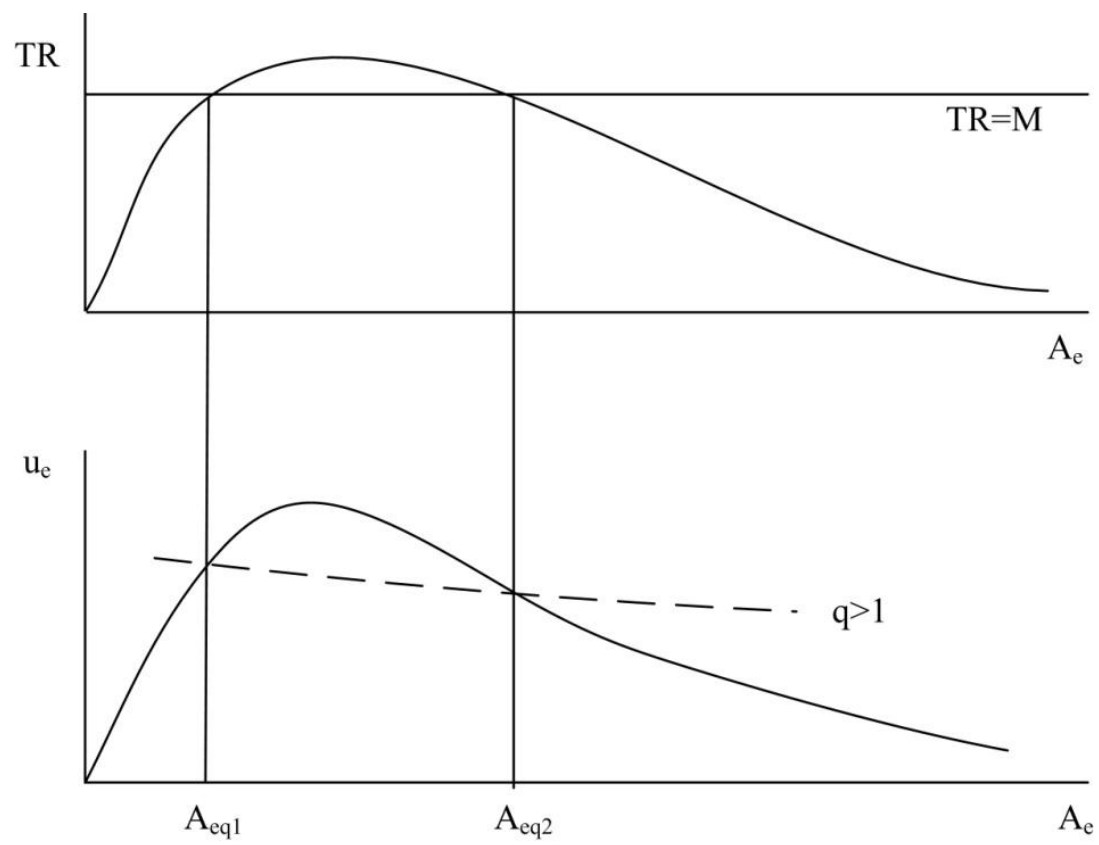

Figure 2. Equilibrium cross-sectional areas (top: equations (5) and (6), bottom: equations: (8) and (9))

Kraus (1998) took the ebb-tidal transport proportional to the amplitude of the bottom shear stress to the power $n / 2$ and the width of the entrance section, $w$. Based on Manning's equation, the bottom shear stress was taken proportional to the velocity squared divided by the one third power of the hydraulic radius $R$ (which is close to the water depth). The resulting expression for the transport is:

$$
T R=k u_{e}^{n} R^{-n / 6} w
$$

Making use of equation (4) it follows:

$$
T R=k u_{e}^{n} A_{e}^{\frac{1-n / 6}{2}}
$$

where the shape factor $\alpha$ is incorporated in $k$.

Comparing to equation (5), it follows $m=1-n / 6$. Substituting in the expression for $q$, it follows that for $n$ between 3 and 5, $q$ varies between 1.03 and 1.20.

Suprijo and Mano (2004) assuming a rectangular cross-section with width w and depth h took

$$
T R=k u_{e}^{5} w / h^{5 / 6}
$$

Making use of equation (4) we derive:

$$
T R=k u_{e}^{5} A_{e}^{-1 / 12}
$$

Comparing to equation (5), it follows $n=5$, while $m=1 / 6$. Substituting in the expression for $q, q=$ 1.02 . 
In conclusion, we state that the $A-P$ relationship should only refer to inlets that are in a stable equilibrium. For a given inlet, this corresponds to the stable equilibrium point in the Escoffier diagram. The velocity curve is a monotonously decreasing function of $A_{e}$, such that the coefficient $q$ in the $A-P$ relationship is larger than 1 . We stress that it is important to assess the value of $q$, because, as shown in Stive et al. (2009), the stable equilibrium cross-sectional area shows appreciable variation for different values of $q$ and accordingly of $C$.

\section{OBSERVATIONS OF THE A-P RELATIONSHIP}

Upfront we state that we object to the clustering of data as done in several of the following references for inlets that are not in similar tidal and morphological environments. Data sets used to determine the constants $C$ and $q$ in equation (1) should ideally consist of inlets that show phenomenological similarity (i.e., have similar values for $k, n, m$ and $\alpha$ for a given inlet situation with mean annual influx of sediment $M$ ), implying that the datasets should be clustered while ensuring:

- similar tide characteristics (form number, amplitude);

- similar grain size and grain density;

- similar wave driven littoral drift;

- similar shape of the cross-section.

However, since we are making a review of the $A-P$ relationship we cannot ignore the widely referenced observations, and will provide these with our comments.

Early basic, well-referenced observations presented in literature are those of O'Brien (1969) and Jarrett (1976). These observations are amply used but do not or not completely fulfil the conditions that we conclude as essential for a reliable conclusion. After reviewing these references we will highlight and comment on observations made since then.

O'Brien (1931) originally determined a relationship between minimum gorge cross-sectional area of an inlet below mean tide level and the tidal prism at spring tide. This relationship was derived from observation of 9 inlets on the Atlantic coast, 18 others on the Pacific coast and one inlet on the Gulf coast in the US. According to O'Brien (1969) the relationship is predominantly valid for Pacific coastal inlets, where a mixed tidal pattern is observed and where typically a strong ebb flow exists between higher-high water and lower-low water. O'Brien (1969) showed that for these 28 US entrances, $C=4.69$ $10^{-4}$ and $q=0.85$ are best-fit values applicable to all entrances when $P$ is measured in cubic meters $\left(\mathrm{m}^{3}\right)$ and $A$ in square meters $\left(\mathrm{m}^{2}\right)$, but when limited to 8 non-jettied entrances he derived $C=1.0810^{-4}$ and $q=1$ as best-fit values. Obviously, O'Brien's data set of 28 inlets does not satisfy the requirement of same littoral drift and tide conditions, and we doubt whether this is the case for the 8 non-jettied entrances.

Probably the most widely referenced dataset is that of Jarrett (1976). He performed a regression analysis on data from 108 inlets along the Pacific, the Atlantic, and the Gulf coast of the US in various combinations in an attempt to determine best-fit equations for tidal inlets on all three coasts of the US and to investigate the change in the relationship for natural (non-stabilized) inlets and for stabilized inlets. In his study, inlets were divided into three main categories according to whether there were no jetties, just one jetty or two jetties. Within each category the inlets were further separated into groups corresponding to the three coasts: Pacific coast, Atlantic coast and Gulf coast. Note that Jarrett (1976) realized that similarity between inlet groups was necessary for a more reliable result. Values of the empirical parameters $(C$ and $q$ ) were determined empirically by plotting values of inlet area versus tidal prism for inlet groups (Table 1). Based on our statement that fits should only be made to inlet data which are phenomenological similar the shaded fields in Table 1 should not be considered reliable. 


\begin{tabular}{|c|c|c|c|c|c|c|}
\hline \multirow[t]{2}{*}{ Location } & \multicolumn{2}{|c|}{ All inlets } & \multicolumn{2}{|c|}{ No jetty or one jetty } & \multicolumn{2}{|c|}{ Two jetties } \\
\hline & $C$ & $q$ & $C$ & $q$ & $C$ & $q$ \\
\hline All inlets & $2.4110^{-4}$ & 0.93 & $3.6510^{-5}$ & 1.04 & $1.4810^{-3}$ & 0.83 \\
\hline Atlantic coast & $6.0410^{-5}$ & 1.02 & $1.9810^{-5}$ & 1.08 & $6.7010^{-4}$ & 0.87 \\
\hline Gulf coast & $9.3010^{-4}$ & 0.84 & $6.9410^{-4}$ & 0.86 & $1.4310^{-3}$ & 0.81 \\
\hline Pacific coast & $4.7510^{-4}$ & 0.88 & $8.8310^{-6}$ & 1.10 & $1.8810^{-3}$ & 0.82 \\
\hline
\end{tabular}

Table 1b. Number of inlets and correlations for the datasets for tidal inlets on US coasts related to Table

1a, where the results in the shaded boxes are not fulfilling our recommendations (data derived and reprocessed from Jarrett, 1976)

\begin{tabular}{|c|c|c|c|c|c|c|}
\hline \multirow[t]{2}{*}{ Location } & \multicolumn{2}{|c|}{ All inlets } & \multicolumn{2}{|c|}{ No jetty or one jetty } & \multicolumn{2}{|c|}{ Two jetties } \\
\hline & \# of inlets & $r^{2}$ & \# of inlets & $r^{2}$ & \# of inlets & $r^{2}$ \\
\hline All inlets & 108 & 0.90 & 71 & 0.92 & 37 & 0.88 \\
\hline Atlantic coast & 59 & 0.92 & 40 & 0.94 & 19 & 0.81 \\
\hline Gulf coast & 24 & 0.87 & 21 & 0.87 & 3 & 0.88 \\
\hline Pacific coast & 25 & 0.92 & 10 & 0.97 & 15 & 0.93 \\
\hline
\end{tabular}

Since the publication of the O'Brien (1969) and Jarrett (1976) datasets many studies published findings for the coefficients $C$ and $q$ for groups of other inlet entrances. We have made an initial scrutiny of these datasets and come to the disappointing conclusion that there exist only two studies that are close to fulfilling our phenomenological similarity condition, viz. Dieckmann et al. (1988), who studied the entrances of the Dutch and German Wadden Sea and Van de Kreeke (1998) who studied the entrances of the Dutch Wadden Sea, It should be stressed here that both these evaluations of the $A-P$ relationship departed somewhat from the original O'Brien definition in that their estimates were based on the mean tidal prism instead of the spring tidal prism. An overview of the fits to these datasets is summarized in Table 2.

\begin{tabular}{|c|c|c|c|c|}
\hline \multicolumn{5}{|c|}{ Table 2 Comparison of further findings for comparable inlet situations } \\
\hline Source & Location (\# of inlets) & $C$ & $q$ & $r^{2}$ \\
\hline Dieckmann et al. (1988) & $\begin{array}{l}\text { Dutch and German Wadden Sea } \\
\text { (37) }\end{array}$ & $3.7210^{5}$ & 0.92 & $?$ \\
\hline Van de Kreeke (1998) & Dutch Wadden Sea (5) & $3.4510^{-4}$ & 0.81 & 0.96 \\
\hline Van de Kreeke (1998) & Dutch Wadden Sea (5) & $6.8010^{-5}$ & 1 & 0.99 \\
\hline
\end{tabular}

From these results we conclude that optimal fits lead to $q$ values that are in a range between 0.81 and 1, which is in clear contrast with our theoretical finding that $q$ should be larger than 1 . If we include the Jarrett (unshaded) dataset this range is between 0.81 and 1.10 , which is more promising but still does not conform to our theoretical findings. We conclude that this is an issue that needs to be resolved and suggest that careful scrutiny of the data sets to check whether they fulfil the phenomenological similarity condition is a first and necessary step towards achieving this. We address this issue in the next section.

\section{RESCRUTINIZING DATA}

The available A-P data from the US Pacific, Atlantic and Gulf coasts (Jarrett, 1976 and Powell, 2003) have been re-scrutinized and categorised following the above mentioned phenomenological similarity criteria, viz. similar tidal range, similar sediment size, similar littoral transport and similar hydraulic radius. All together, some 20 different categories were considered and $A-P$ relationships were obtained for each category. Generally, high correlations were found between the stable inlet predicted by each A-P relationship and the corresponding data (see Tables 3, 4, 5 and 6). However, if we 
compare the correlations with Jarrett's dataset in Table $1 \mathrm{~b}$, they are not significantly better, in some cases they are even worse. The latter may be a result of insufficient data points for a particular category.

Finally, we point out that only in a small number of categories (Jarrett's data for a small and a large hydraulic radius; see Figure 3 for the example large hydraulic radius) does the $q$ value associated with the $A-P$ relationship exceed unity as suggested by the theoretical derivations. In the majority of categories the $q$ value associated with the $A-P$ relationship does not exceed unity. This is truly disappointing and we have no physical explanation for this. Clearly this is an issue that remains unresolved.

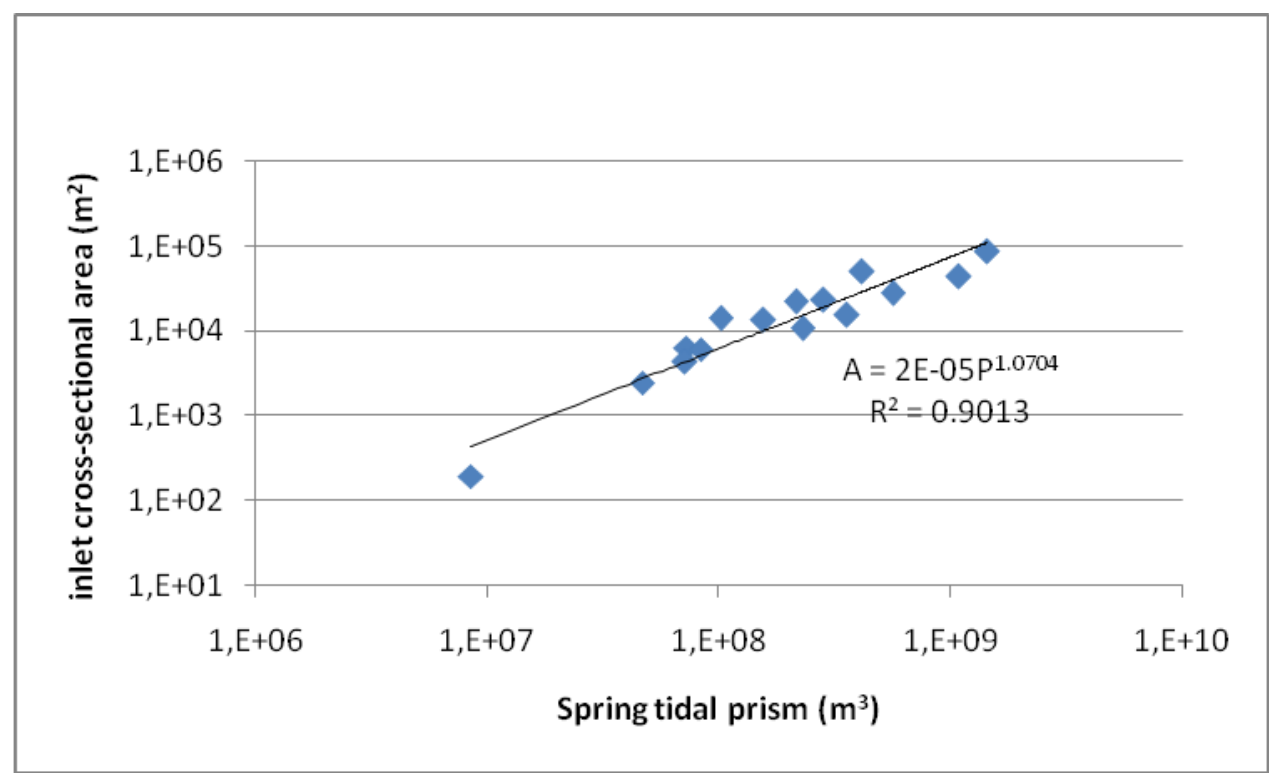

Figure 3. The A-P relationship for inlets with hydraulic radii exceeding $>30$ (large hydraulic radius category). Note the $q$ value $(>1)$ and the high correlation coefficient.

\begin{tabular}{|l|l|l|l|}
\hline \multicolumn{4}{|c|}{ Table 3 A-P relationship based on diurnal tidal range in } \\
metric units
\end{tabular}

\begin{tabular}{|c|c|c|c|}
\hline \multicolumn{4}{|c|}{$\begin{array}{l}\text { Table } 4 \text { A-P relationship based on medium sediment size in } \\
\text { metric units }\end{array}$} \\
\hline & $\mathrm{C}$ & $q$ & $r^{2}$ \\
\hline Powell fine sand & $3 \times 10^{-4}$ & 0.91 & 0.91 \\
\hline Powell medium sand & $1.7 \times 10^{-3}$ & 0.81 & 0.82 \\
\hline Powell course sand & 1.37 & 0.39 & 0.34 \\
\hline Jarret silt & $1.5 \times 10^{-3}$ & 0.94 & 0.94 \\
\hline Jarret very fine sand & $9 \times 10^{-4}$ & 0.84 & 0.78 \\
\hline Jarret fine sand & $9 \times 10^{-5}$ & 0.99 & 0.95 \\
\hline Jarret medium sand & $6 \times 10^{-5}$ & 1 & 0.83 \\
\hline Jarret course sand & $8 \times 10^{-5}$ & 1 & 0.99 \\
\hline
\end{tabular}




\begin{tabular}{|l|l|l|l|}
\hline Table 5 A-P relationship based on annual net longshore transport in metric \\
units \\
\hline & $\mathrm{C}$ & $\mathrm{q}$ & $\mathrm{r}^{2}$ \\
\hline Powell low longshore transport & $2.1 \times 10^{-3}$ & 0.79 & 0.88 \\
\hline Powell medium longshore transport & $1.2 \times 10^{-2}$ & 0.69 & 0.56 \\
\hline Jarret low longshore transport & $3 \times 10^{-4}$ & 0.92 & 0.92 \\
\hline Jarret medium longshore transport & $8 \times 10^{-5}$ & 1 & 0.89 \\
\hline Jarret large longshore transport & $2 \times 10^{-4}$ & 0.92 & 0.83 \\
\hline
\end{tabular}

\begin{tabular}{|l|l|l|l|}
\hline \multicolumn{4}{|c|}{ Table 6 A-P relationship based on hydraulic radius in metric } \\
units \\
\hline & C & $q$ & $r^{2}$ \\
\hline Powell small radius & $7 \times 10^{-4}$ & 0.85 & 0.95 \\
\hline Jarrett small radius & $3 \times 10^{-5}$ & 1.06 & 0.85 \\
\hline Jarrett medium radius & $2 \times 10^{-4}$ & 0.94 & 0.81 \\
\hline Jarrett large radius & $2 \times 10^{-5}$ & 1.07 & 0.90 \\
\hline
\end{tabular}

We finally note that we have succeeded in deriving a new multi-parameter relationship to predict the stable inlet cross-section via multiple regression analysis across all phenomenological categories. The relationship is of the form:

$$
A=C P^{q} x^{l} y^{m} z^{n} \ldots
$$

where $x, y, z$ etc represent the various phenomena identified above.

A comparison with available data indicates that the new relationship produces more accurate stable inlet cross-section predictions than previous empirical relationships derived by Jarrett (1976) and Powell et al (2006). However, we do not consider this result of high value since it is of little generic use. What is an interesting result from this exercise is that the most important parameters determining the inlet cross-sectional area for the US coast appear to be the spring tidal prism and the cross-sectional geometry (hydraulic radius or width and depth).

\section{CONCLUSIONS}

We have reviewed the well-known empirical relationship (equation 1) expressing the equilibrium cross-sectional area of tidal inlet entrances as a function of the tidal prism $P$ under forcing of the littoral drift. Our theoretical investigations indicate that a unique $A-P$ relationship should only be expected for clusters of inlets that are phenomenological similar (i.e. fairly similar hydrodynamic and morphological conditions), and that the exponent $q$ in the $A-P$ relation should be larger than 1 . However, relevant published data available to date do not clearly support this theoretical finding. A re-analysis of the available data sets by Stive et al. (2009) indicated that they may not be sufficiently reliable to verify our theoretical finding with regard to $q>1$ due to the violation of the condition of phenomenological similarity, and possibly also due to violating the initial definitions given by O'Brien (1931) in estimating the tidal prism. The resolution of this issue is important because slightly different values of $q$ result in significantly variable values for the equilibrium cross-sectional area of the tidal entrance. This may have significant implications in determining the true stable equilibrium entrance cross-sectional area. Here we present a re-analysis of the available data with a focus on determining the phenomenological dependencies of the $A-P$ relationship. The available $A-P$ data from the US Pacific, Atlantic and Gulf coasts (Jarrett, 1976 and Powell, 2003) have been re-scrutinized and categorized following the above mentioned phenomenological similarity criteria, viz. similar tidal range, similar sediment size, similar littoral transport and similar hydraulic radius. All together, some 20 different categories were considered and $A-P$ relationships were obtained for each category. Generally, high correlations were found between the stable inlet predicted by each A-P relationship and the corresponding data. However, only in a limited number of categories were the correlations significantly better than the correlations for the complete datasets. Finally, we point out that only in a number of categories did the $q$ value associated with the $A-P$ relationship exceed unity as suggested by the theoretical derivations. In the majority of categories the $q$ value associated with the $A-P$ relationship does not exceed unity. 


\section{REFERENCES}

Dieckmann, R., Osterhun, M., Partenscky, H.W., 1988. A comparison between German and North American tidal inlets. Proceedings of the $21^{\text {st }}$ International Conference on Coastal Engineering, ASCE, 2681-2691

Escoffier, F.F., 1940. The stability of tidal inlets. Shore and Beach 8 (4), $111-114$

Gerritsen, F., and Louters, T, 1990. Morphological stability of inlets and channels in the Western Wadden sea. Rijkswaterstaat, Report GWAO-90.019.

Hume, T.M. and Herdendorf, C. E., 1990. Morphologic and Hydrologic Characteristics of Tidal Inlets on a Headland Dominated, Low Littoral Drift Coast, Northeastern New Zea1and, Proceedings Skagen Symposium (2-5 Sep. 1990), Journal of Coastal Research Special Issue 9: 527-563.

Hume, T.M., Herdendorf, C. E., 1993. On the use of empirical stability relationships for characterising estuaries. Journal of Coastal Research 9 (2), 413- 422.

Jarrett, J.T., 1976. Tidal prism - inlet area relationships. GITI report no. 3. Coastal Engineering Research Center, US Army Corps of Engineers, Fort Belvoir, VA, USA

Kraus, N.C., 1998. Inlet cross-section area calculated by process-based model. Proceedings International Conference on Coastal Engineering. ASCE, Reston, VA, pp 3265-3278

LeConte, L.J., 1905. Discussion on the paper, "Notes on the improvement of river and harbor outlets in the United States" by D. A. Watt, paper no. 1009. Trans. ASCE 55 (December): 306-308

O’Brien, M.P., 1931. Estuary and Tidal Prisms Related to Entrance Areas. Civil Eng. 1 (8): 738-739.

O'Brien, M.P., 1969. Equilibrium flow areas of inlets on sandy coasts. J Waterway Port Coast Ocean Eng 95 (1): 43-52

Powell, M.A., R.J. Thieke and A.J. Mehta, 2006. Morphodynamic relationships for ebb and flood delta volumes at Florida's entrances. Ocean Dynamics 56: 295-307.

Powell M.A., 2003. Ebb shoal and flood shoal volumes on the coasts of Florida: St Marys entrance to Pensacola Pass. Masters theses University of Florida.

Riedel, H.P., and Gourlay, M.R., 1980. Inlets/Estuaries Discharging into Sheltered Waters. Proceedings International Conference on Coastal Engineering, ASCE Press, NY, 2550-2562.

Shigemura, T., 1980. Tidal prism - throat area relationships of the bays of Japan. Shore and Beach, 48 (3): $30-35$

Stive M.J.F., Kreeke J. van de, Nghiem T.L., Tran Thanh T., Ranasinghe, R.W.M.R.J.B., 2009. Empirical relationships between inlet cross-section and tidal prism: a review. In M Mizuguchi \& S Sato (Eds.), Proceedings of Coastal Dynamics 2009. Impacts of human activities on dynamic coastal processes (pp. 1-10). Tokyo, Japan: World Scientific Publishing Co. Pte. Ltd.

Suprijo, T. and Mano A., 2004. Dimensionless parameters to describe topographical equilibrium of coastal inlets. Proc . $29^{\text {th }}$ ICCE, ASCE: 2531-2543

Van de Kreeke, J., 1985. Stability of tidal inlets; Pass Cavallo, Texas. Estuarine Coastal Shelf Science, $21: 33-43$

Van de Kreeke, J., 1990. Stability of a two-inlet bay system. Coastal Engineering, 14: 481-497

Van de Kreeke, J., 1992. Stability of tidal inlets; Escoffier's analysis. Shore and Beach, 60 (1): 9-12

Van de Kreeke, J., 1998. Adaptation of the Frisian inlet to a reduction in basin area with special reference to the cross-sectional area of the inlet channel. In Dronkers, J. and Scheffers , M.B.A.M. (Eds). Proc. PECS conference: $355-362$

Van de Kreeke, J., 2004. Equilibrium and cross-sectional stability of tidal inlets: application to the Frisian Inlet before and after basin reduction. Coastal Engineering, 51: 337-350

Yanez, M. A., 1989. Stability of a double -inlet bay system; Marco Island, FL, USA. Master's thesis, RSMAS, University of Miami, pp 67 